\title{
Tyrosine kinase inhibitors in breast cancer (Review)
}

\author{
GEORGE IANCU ${ }^{1,2}$, DRAGOS SERBAN ${ }^{3,4}$, CRISTINEL DUMITRU BADIU ${ }^{3,5}$, \\ CIPRIAN TANASESCU ${ }^{6}$, MIHAI SILVIU TUDOSIE ${ }^{7,8}$, CORNELIU TUDOR $^{3}$, \\ DANIEL OVIDIU COSTEA $^{9,10}$, ANCA ZGURA ${ }^{11}$, RALUCA IANCU ${ }^{12,13}$ and DANUT VASILE ${ }^{3,14}$
}

\begin{abstract}
${ }^{1}$ Department of Obstetrics and Gynecology, Faculty of Medicine, 'Carol Davila' University of Medicine and Pharmacy, 020021 Bucharest; ${ }^{2}$ Department of Gynecology, 'Filantropia' Clinical Hospital, 011132 Bucharest;

${ }^{3}$ Department of General Surgery, Faculty of Medicine, 'Carol Davila' University of Medicine and Pharmacy, 020021 Bucharest; ${ }^{4}$ Fourth Department of General Surgery, Emergency University Hospital, 050098 Bucharest;

${ }^{5}$ Department of General Surgery, 'Prof. Dr. Bagdasar Arseni' Emergency Clinical Hospital, 041915 Bucharest;

${ }^{6}$ Third Clinico-Surgical Department, Faculty of Medicine, 'Lucian Blaga' University, 550169 Sibiu; ${ }^{7}$ Department of

Orthopedia and Intensive care, Faculty of Medicine, 'Carol Davila' University of Medicine and Pharmacy, 020021 Bucharest; ${ }^{8}$ ICU II Toxicology, Clinical Emergency Hospital, 014461 Bucharest; ${ }^{9}$ Department of General Surgery, Faculty of Medicine, 'Ovidius' University, 900470 Constanta; ${ }^{10}$ First Surgery Department, Emergency County Hospital, 900591 Constanta; ${ }^{11}$ Department of Oncology Radiotherapy, Institute of Oncology 'Prof. Dr. Trestioreanu', 022328 Bucharest; ${ }^{12}$ Department of ENT-Opthalmology, Faculty of Medicine, Carol Davila' University of Medicine and Pharmacy, 020021 Bucharest; ${ }^{13}$ Department of Ophthalmology and

${ }^{14}$ First Department of General Surgery, Emergency University Hospital, 050098 Bucharest, Romania
\end{abstract}

Received August 20,2021; Accepted September 20, 2021

DOI: $10.3892 / \mathrm{etm} .2021 .11037$

\begin{abstract}
Anti-epidermal growth factor receptor (EGFR)-targeted therapy has been intensely researched in the last years, motivated by the favorable results obtained with monoclonal antibodies in HER2-enriched breast cancer (BC) patients. Most researched alternatives of anti-EGFR agents were tyrosine kinase inhibitors (TKIs) and monoclonal antibodies. However, excluding monoclonal antibodies trastuzumab and pertuzumab, the remaining anti-EGFR molecules have exhibited disappointing results, due to the lack of specificity and frequent adverse side effects. TKIs have several advantages, including reduced cardiotoxicity, oral administration and favorable penetration of blood-brain barrier for brain metastatic BC. Lapatinib and neratinib and recently pyrotinib (approved only in China) are the only TKIs from dozens of molecules researched over the years that were approved to be
\end{abstract}

Correspondence to: Dr Dragos Serban, Department of General Surgery, Faculty of Medicine, 'Carol Davila' University of Medicine and Pharmacy, 37 Dionisie Lupu Street, 020021 Bucharest, Romania E-mail: dragos.serban@umfcd.ro

Abbreviations: EGFR, epidermal growth factor receptors; TKIs, tyrosine kinase inhibitors; NSCLC, non-small cell lung cancer; BC, breast cancer

Key words: breast cancer, tyrosine kinase inhibitor, anti-EGFR agents, lapatinib, neratinib, pyrotinib used in clinical practice with limited indications, in a subset of $\mathrm{BC}$ patients, single or in combination with other chemotherapy or hormonal therapeutic agents. Improved identification of $\mathrm{BC}$ subtypes and improved characterization of aggressive forms (triple negative BC or inflammatory BC) should lead to advancements in shaping of targeted agents to improve the outcome of patients.

\section{Contents}

1. Introduction

2. Tyrosine kinase inhibitors (TKIs) used in breast cancer (BC) therapy

3. Limitations and challenges of TKI use in BC chemotherapy

4. Conclusions

\section{Introduction}

The epidermal growth factor receptor (EGFR) family consists of four categories of tyrosine kinase receptors including ErbB1 (HER1), ErbB2 (HER2), ErbB3 (HER3) and ErbB4 (HER4) (1). Abnormal activation of these kinases results in avoidance of apoptosis, excessive cell growth and angiogenesis in epithelial cancer (2). HER2 is activated after dimerization following ligand attachment; dimerization starts intracellular auto-phosphorylation of tyrosine residues and initiates the signaling pathways of cell proliferation. The mitogenic activity is initiated by homo- or hetero-dimerization of HER proteins; heterodimers generate 
more potent signals, with the HER2-HER3 heterodimer as the most potent stimulator (3).

Optimal results for BC therapy were obtained with HER2 blockade that became a standard targeted therapy after HER2 overexpression was demonstrated in up to $25 \%$ of BC (4). The implication of other HER receptors, apart from HER2, in cell signaling and proliferation through heterodimerization, has led researchers to the concept of blocking other HER receptors as well, for improved anti-proliferative results (3-5). However, the remaining EGFR targets are still under investigation with non-satisfactory results to date.

One of the challenges of anti-EGFR targeted therapy is the triple negative breast cancer (TNBC) subtype. TNBC has been revealed to have frequently overexpressed EGF receptors (4). Immunohistochemical analysis of the overexpression of EGFR in TNBC varies widely in literature, roughly between 10 and $75 \%$ (6-8). Lack of hormonal receptors and HER-2 expression renders TNBC as one of the most resistant BC subtypes to conventional therapy.

Specific anti-EGFR therapies include compounds acting as TKIs such as gefitinib, erlotinib, afatinib, lapatinib, osimertinib, neratinib, canertinib, tucatinib and pyrotinib as well as monoclonal antibodies (mAb) trastuzumab, pertuzumab, cetuximab, panitumumab, and necitumumab. These are used in a variety of cancers, most frequently in lung, head and neck and pancreatic cancer (9).

The aim of this review was to present the TKI agents of potential use in BC therapy, documenting the specific mechanism of action, indications and possible side effects. In the present review, the latest data from international clinical trials concerning the indications, combinations with other agents and results in treating different $\mathrm{BC}$ subtypes were collected. A compressive search was performed on PubMed, Google Scholar and Web of Science, using the terms 'breast cancer', and 'tyrosine kinase inhibitors' or 'gefitinib' or 'erlotinib' or 'afatinib' or 'lapatinib' or 'tucatinib' or 'neratinib' or 'canertinib' or 'pyrotinib'. All clinical studies in English, published until 2019, regarding the use of tyrosine kinase inhibitors in patients with breast cancers were included. Handsearching was performed for relevant additional studies in the reference list of the systematic reviews on the topic. Articles providing insufficient data regarding the outcomes and side-effects of the therapy were excluded.

TKIs block abnormal signal transduction pathways necessary for cell proliferation and growth. Most of the TKIs inhibit multiple pathways in the signaling chains. Anti-EGFR agents target tyrosine kinase receptor that plays an important role in numerous types of cancer. There are numerous agents undergoing investigation in preclinical and clinical trials (10).

\section{TKI used in BC therapy}

Anti-EGFR drugs inhibiting tyrosine kinase currently used in clinical practice include gefitinib [non-small cell lung cancer (NSCLC)], erlotinib (NSCLC, pancreatic cancer), afatinib (NSCLC), and osimertinib (NSCLC), while for BC therapy there are lapatinib, neratinib and pyrotinib currently approved, mostly in combination with other agents $(9,10)$.

Mechanisms of action. Stimulation of tyrosine kinases leads to altering of the cell cycle, angiogenesis and lack of apoptosis in epithelial malignant cells (2). Mutation of EGFR enhances sensitivity of the receptor to the ligand and plays an important role in different types of cancer, with lung cancer as the most studied (11).

TKIs block the ATP-binding site and inhibit EGFR kinase activity. They are widely used for NSCLC with markedly favorable results. However, resistance occurs in most patients treated on a long-term basis, usually through secondary mutations (12). Mechanisms of resistance are divided into several categories including primary resistance, acquired resistance and persistent resistance. The mechanisms are multiple and include secondary mutation of the tyrosine kinase, gene amplification and overexpression of the protein kinase, activation of different signaling pathways, overexpression of kinases downstream of the kinase, epigenetic mechanisms, as well as lower intracellular drug concentrations (for example, mechanisms such as extracellular sequestration of the inhibitor by binding to $\alpha$ acid glycoprotein, decreased expression or activity of drug influx pumps, and increased expression or activity of drug efflux pumps) (13). Occasionally, resistance and sensitivity to chemotherapy are influenced by mutation in p53 genes, but mutant p53 effects have to be interpreted in the clinical context and not isolated (14).

\section{Clinical trials}

Gefitinib. Most TKIs are still being evaluated in preclinical trials and in phase I or II clinical trials. They have been used as monotherapy or in combination with other agents already in clinical use. Unfortunately, results have been often disappointing when efficacy was assessed in the BC patient population. Clinical trials in advanced breast cancer (ABC) revealed low clinical response rates in patients treated with gefitinib alone or in combination with other agents (15-17). Baselga et al surmised that the low response rate was due to the lack of EGFR dependence of the BC population studied and not to the lack of inhibition of receptors itself (15). At a dose of $500 \mathrm{mg} /$ day of gefitinib, most frequent adverse events (AEs) were diarrhea, skin toxicity (rash and erythema) and asthenia. Gefitinib was well tolerated and the majority of side effects were grade 1 or 2 gastrointestinal and skin reactions. Skin rashes and diarrhea were the most common grade 3 and 4 side effects. A total of 9 patients underwent dose interruptions or reductions due to skin toxicity (15). Another study revealed a $13 \%$ rate of discontinuation due to AEs in the gefitinib plus anastrozole group compared with a rate of $2 \%$ in the anastrozole only group (17). Green et al found a clinical benefit rate (CBR) of gefitinib in an adjuvant setting in $\mathrm{ABC}$ of $11 \%$ in a hormone-resistant population and of $7.7 \%$ in a hormone-negative population; the study ended prematurely due to low response rate and high toxicity (20\% of patients had dose reduction and $46 \%$ had to stop the study medication). Most frequently encountered grade 3 and 4 AEs were diarrhea (17\%) and skin rashes (12\%) (16).

When studies were performed on a selected EGFR-positive BC population, improved outcomes (partial response) were observed when gefitinib alone or in combination (anastrozole) was administered in a neoadjuvant or metastatic setting $(18,19)$. Polychronis et al identified a mean reduction in the proliferation-related Ki67 index of $98 \%$ vs. $92.4 \%$ for combined anastrozole and gefitinib compared with gefitinib 
alone, respectively $(\mathrm{P}=0.0054)$. Most commonly encountered side effects were gastrointestinal and skin-related toxicity, of which $5.4 \%$ were grade 3 or 4 (18). A randomized clinical trial assessing gefitinib plus anastrozole or plus fulvestrant in metastatic BC revealed similar response rates between the two therapies, but no clear advantage when compared with endocrine therapy alone. The toxicity was in general greater with the addition of gefitinib (20). When compared in a randomized trial, in a neoadjuvant setting, gefitinib revealed a significantly higher pathologic complete response (pCR) rate in TNBC when compared with non-TNBC. Tumor response rates were similar in the two groups, while hematological toxicity was significantly higher in the gefitinib arm (21). A recent trial assessing the benefit of adding gefitinib to anastrozole was terminated prematurely due to lack of improvement of progression-free survival (PFS) of patients with hormone receptor-positive BC; gefitinib-related skin and gastrointestinal toxicities caused premature therapy interruption in almost $30 \%$ of patients. The most frequent grade 3 and 4 adverse event was diarrhea in $11 \%$ of patients (22).

Erlotinib. A multicenter phase II trial revealed little efficacy of erlotinib in an unselected population of patients with locally advanced or metastatic BC that were previously treated with different cytotoxic agents (3\% partial response, with a median time to progression of 43 days) Most frequent toxicities were grade 3/4 nausea, diarrhea and vomiting ( 4-6\% each) and acne $(7.4 \%)$. Pancreatitis and fever were the two serious AEs reported (23). Erlotinib was found to increase apoptosis of $\mathrm{BC}$ cells in preclinical trials, in tumors with increased EGFR expression (basal-like BC) (24). Although it was revealed to inhibit TNBC in preclinical trials, inhibition of metastasis was accompanied by other effects due to its lack of specificity of kinase inhibitor effects; toxicity of study medication was also high (25). In a neoadjuvant clinical setting, erlotinib was revealed to be efficient in patients with estrogen receptor-positive tumors, but with little effect on triple negative or HER2-positive BC. Most common grade 1 and 2 side effects were rash and diarrhea (60 and $24 \%$ of patients, respectively). A total of 6 patients were excluded due to medication toxicity effects (14.6\%) (26).

Afatinib. Afatinib irreversibly blocks HER family, acting as a potent oral agent on HER1, HER2 and HER4 receptors. However, afatinib has demonstrated little efficacy in HER2-positive BC. It was evaluated in a randomized phase III trial on metastatic BC-overexpressing HER2 patients who had progressed on one previous trastuzumab regimen. Afatinib was compared with trastuzumab, both in combination with vinorelbine, and it was revealed to lead to disappointing results, and thus, trastuzumab-based therapy remained the mainstay of therapy in this population. The study was prematurely ended because the risk-benefit assessment revealed no advantage of afatinib therapy. Median follow-up was 9.3 months; PFS was 5.5 months for the afatinib group vs. 5.6 months for the trastuzumab group. Drug-related AEs were grade 3/4 neutropenia (56\%), leucopenia (19\%) and diarrhea (18\%) (27).

Afatinib did not show any significant benefit for patients with HER2-positive tumors and brain metastasis (patients benefited in $30 \%$ of cases administered afatinib alone; the difference vs. the investigator's choice was non-significant, $\mathrm{P}=0.37$ ); the regimen containing afatinib was less well tolerated (more frequent grade 3 or 4 diarrhea or neutropenia) (28). However, the penetration rate of afatinib in the cerebrospinal fluid appears to be favorable enough to achieve clinical response in patients with central nervous system metastases (29).

The DAFNE trial evaluated efficacy of combined treatment afatinib and trastuzumab, followed by taxane/anthracycline chemotherapy in HER2-enriched BC; pathological complete response rate was $49.2 \%$, similar with other anti-HER 2 combinations, but below the expected rate. Patients experienced grade $3 / 4$ non-hematologic toxicity including diarrhea (7.7\%), increased creatinine (4.6\%) and infection (4.6\%) (30). The role of afatinib in HER2-negative BC was researched in the randomized phase II trial, TRIO-020. The comparison was performed between letrozole and afatinib vs. letrozole alone. Unfortunately, the trial was prematurely terminated, due to financial reasons and no results were published.

Lapatinib. Lapatinib is a reversible inhibitor of HER1 and HER 2 receptors. It increased the pCR rate when combined with trastuzumab in HER2-enriched BC patients, compared with trastuzumab alone in a neoadjuvant setting. However, higher toxicity was noted for combined administration. Patients on lapatinib experienced more often diarrhea, skin toxicity, infections and hepatic toxicity (31). The NeoALTTO trial revealed significantly higher pCR rates after the use of combined trastuzumab and lapatinib in a neoadjuvant setting for HER2-positive early BC than either agent alone (pCR $51.3 \%$ vs. $29.5 \%$ for combined treatment vs. trastuzumab alone respectively; $\mathrm{P}=0.0001)$. However overall survival $(\mathrm{OS})$ and event-free survival were similar between groups $(\mathrm{P}=0.19$ for combined treatment vs. trastuzumab for OS) $(32,33)$. Toxicity was significant. For the lapatinib group, $65 \%$ of the discontinuations in the neoadjuvant and $31 \%$ in the adjuvant phase were due to AEs. Most common grade 3 and 4 AEs in the lapatinib group were diarrhea $25 \%$, hepatic toxicity $22 \%$ and neutropenia $17 \%$ (33).

EORTC, a phase II trial demonstrated a modest increase in pCR in HER2-positive BC when lapatinib and trastuzumab were used in a neoadjuvant setting as a double blockade of HER 2 plus chemotherapy (56\% for combined treatment, 52\% for trastuzumab and $36 \%$ for lapatinib) (34).

Although lapatinib plus trastuzumab increased pCR and improved outcomes for HER2-positive BC in a neoadjuvant setting, in an adjuvant setting their association did not increase disease-free survival (DFS) rates and increased toxicity ( $\mathrm{P}=0.048$; with significantly more side effects for the group receiving lapatinib) (ALTTO trial). For one year, the treatment with adjuvant trastuzumab remained the standard recommendation for HER2-enriched metastatic BC (35).

Currently, lapatinib is approved by Food and Drug Administration (FDA) for BC treatment in combination with capecitabine or letrozole. Geyer et al compared lapatinib and capecitabine with capecitabine alone in locally advanced BC and metastatic $\mathrm{BC}$ and found a median time to progression of 8.4 months for combined treatment, significantly higher than the 4.4 months in the capecitabine alone group (36). FDA approved lapatinib (Tyverb ${ }^{\circledR}$ ) to be used in combination with letrozole in hormone receptor- and HER2-positive BC because 
it was revealed to increase PFS, objective response rate and CBR in the multinational large randomized trial, EGF30008 (PFS, 8.2 vs. 3 months for lapatinib and letrozole vs. letrozole alone respectively) (37-39). Lapatinib can also be combined with trastuzumab, for HER2-positive postmenopausal patients with hormone receptor-positive disease; however, only PFS increase was demonstrated [hazard ratio $(\mathrm{HR})=0.73, \mathrm{P}=0.008$ ], without any benefit on $\mathrm{OS}(\mathrm{P}=0.106)(40,41)$.

Neratinib. Neratinib is an irreversible pan-HER TKI (acting on HER1, HER2 and HER4). In combination with paclitaxel in patients with HER2-positive metastatic BC in a phase I/II clinical trial, it demonstrated a high rate of response $(73 \%$ overall response rate) although with a higher toxicity rate, requiring dose reduction or symptomatic medication (42). A multicenter randomized phase III trial of patients with HER2-positive early-stage BC revealed promising results: after 12 months of treatment, neratinib improved the 2-year invasive DFS rate compared with the placebo, when administered after trastuzumab and chemotherapy in an adjuvant setting (93.9 vs. $91.6 \%$ for neratinib and the placebo, respectively; $\mathrm{P}=0.0091$ ) (ExteNEt trial). Most frequent grade 3 or 4 AEs in the neratinib group were digestive including diarrhea, vomiting and nausea (43). Neratinib was approved in 2017 by the FDA to be administered in an adjuvant setting in patients with HER2-positive BC who finished one year adjuvant trastuzumab, to increase the DFS rate. The recommended dosage for neratinib is $240 \mathrm{mg}$ once a day, orally.

Canertinib. Canertinib (CI-1033) is a pan-HER tyrosine kinase irreversible inhibitor that exerts its anticancer effects on tumors overexpressing HER1, HER2 and HER4; it is still assessed in early-phase trials of HER2-positive metastatic BC. However, in a previous study, the results were modest and high doses were followed by unacceptable toxicity (44).

Tucatinib. Tucatinib is an oral TKI selectively acting on HER2, with reduced effects of EGFR blockade and a favorable toxicity profile, investigated for its role in $\mathrm{BC}$ progressing under current therapies. HER2-positive BC patients with metastatic disease progressing under currently approved HER2-targeted therapies have limited therapeutic options.

It has been evaluated in a phase I trial in combination with trastuzumab and capecitabine, with favorable antitumor efficacy in metastatic BC, brain metastases included. Cited side effects were diarrhea, nausea and vomiting and palmo-plantar erythrodysesthesia, but grade 3 or higher AEs were observed only in $\sim 10 \%$ of patients. Tucatinib in combination with trastuzumab and capecitabine exhibited a favorable antitumor effect with objective response observed in $83 \%$ of patients for the tucatinib-capecitabine combination, $40 \%$ in the tucatinib-trastuzumab combination and $61 \%$ in the tucatinib, capecitabine and trastuzumab combination (45). The PFS rate was $33.1 \%$ compared with $12.3 \%$ in the tucatinib-combination group vs. the placebo-combination group respectively $(\mathrm{P}<0.001)$, while the OS rate at the 2-year follow-up was 44.9 vs. $26.6 \%$ in the tucatinib-combination group and the placebo-combination group, respectively $(\mathrm{P}=0.005)$. Tucatinib efficacy in the brain metastases subgroup was even more evident with a PFS at 1-year follow-up of $24.9 \%$ in the tucatinib-combination group compared with $0 \%$ in the placebo-combination group $(\mathrm{P}<0.001)$. The combination of tucatinib with trastuzumab and capecitabine in HER2-positive metastatic BC provided clear benefits in the PFS and OS rates when compared with the placebo combination (46).

Pyrotinib. Pyrotinib (SHR1258) (Irene ${ }^{\circledR}$ ) is an irreversible pan-ErbB TKI acting on HER1, HER2 and HER4, blocking the cell cycle in G1-phase and inhibiting tumor growth (47). It was approved by the Chinese State Drug Administration as a combination regimen with capecitabine for patients with HER2-positive advanced or metastatic BC and those already treated with anthracycline or taxane chemotherapy. In vitro research trials of pyrotinib in combination with CDK4/6 inhibitor, palbociclib, revealed synergistic efficacy in inhibiting cell proliferation on human cell lines of HER2-positive $\mathrm{BC}$ (48).

Most of the clinical trials involving pyrotinib are currently underway, and are mainly phase I and II trials (49). There are currently two phase I trials with pyrotinib completed, both in China, with a total number of 78 patients (NCT01937689, is a study of pyrotinib in patients with HER2-positive ABC. NCT02361112, is a study evaluating pyrotinib in combination with capecitabine in patients with HER2-positive metastatic BC (BLTN-Ic).

The oral dose of pyrotinib was increased from 80 to $480 \mathrm{mg}$ once daily in the first phase I trial, in metastatic HER2-positive BC patients without prior TKI treatments; the maximum tolerated dose was $400 \mathrm{mg}$. Common adverse effects were diarrhea, vomiting, oral ulcerations, asthenia, leukopenia (44\% experienced diarrhea and the rest of the events were each $\sim 10-13 \%$ ). The median PFS was 35.4 weeks and the overall response rate was $50 \%$ in the study population; higher response rates were observed in the trastuzumab naïve patients (47).

A phase II trial evaluated the combination of pyrotinib with capecitabine compared with lapatinib plus capecitabine in a population of metastatic or recurrent HER2-positive BC patients. The dose of pyrotinib administered was $400 \mathrm{mg}$ orally once daily, combined with $1,000 \mathrm{mg} / \mathrm{m}^{2}$ capecitabine twice daily; most frequent AEs were diarrhea, palmoplantar erythrodysesthesia, vomiting and nausea. The objective response rate was $78.5 \%$ for pyrotinib and capecitabine compared with $57.1 \%$ for lapatinib and capecitabine, while the PFS rate was 18.1 months compared with 7.0 months, respectively (50). The combination pyrotinib and capecitabine exhibited an excellent antitumor effect on HER2 recurrence and metastatic breast carcinoma.

Pyrotinib combined with capecitabine was compared in a phase III trial with placebo and capecitabine in metastatic HER2-positive BC patients treated previously with taxanes and trastuzumab. The median PFS rate for the pyrotinib group was 11.1 months compared with 4.1 months for the placebo group (51).

Pyrotinib combined with trastuzumab, paclitaxel and cisplatin exhibited favorable results (pCR) in HER2-positive locally advanced BC in published case reports (52), while study protocols for randomized trials including pyrotinib are currently under development (53). Clinical trials assessing the effects of TKIs in breast cancer are included in Table I. 
Table I. Clinical trials assessing the effects of tyrosine kinase inhibitors in BC.

\begin{tabular}{|c|c|c|c|c|}
\hline First author (Refs.) & Year & Phase & Disease stage, regimen used & Outcome \\
\hline \multicolumn{5}{|l|}{ A, Gefitinib (Gt) } \\
\hline Baselga et al (15) & 2005 & II & -ABC, Gt monotherapy & -Reduced clinical antitumor activity \\
\hline Green et al (16) & 2009 & II & $\begin{array}{l}\text {-ABC, hormone-resistant/negative, } \\
\text { Gt monotherapy }\end{array}$ & -Low CBR $11 \%$ vs. $7.7 \%$ \\
\hline Smith et al (17) & 2007 & II & -EBC, Gt + anastrozole vs. anastrozole & -No additional clinical effect \\
\hline Polychronis et al (18) & 2005 & II & $\begin{array}{l}\text { Primary } \mathrm{BC}, \mathrm{ER}^{+}, \mathrm{HER} 2^{+} \text {, neoadjuvant, } \\
\text { anastrozole }+\mathrm{Gt} \text { vs. Gt }\end{array}$ & $\begin{array}{l}\text { Significant mean reduction of } \\
\text { proliferation-related Ki67 index } \\
(98 \% \text { vs. } 92.4 \%)\end{array}$ \\
\hline Cristofanilli et al (19) & 2010 & II & $-\mathrm{MBC}, \mathrm{ER}^{+}, \mathrm{Gt}+$ anastrozole vs. anastrozole & -Increase of PFS by adding Gt \\
\hline Carlson et al (20) & 2012 & II & $-\mathrm{MBC}, \mathrm{Gt}+$ anastrozole/fulvestrant & $\begin{array}{l}\text { Similar CBR, response rates similar } \\
\text { with Gt or endocrine therapy alone }\end{array}$ \\
\hline Bernsdorf et al (21) & 2011 & II & EBC, neoadjuvant, TNBC vs. non-TNBC & Higher pCR in TNBC, higher toxicity \\
\hline Tryfonidis et al (22) & 2016 & II & $\begin{array}{l}-\mathrm{ABC} \text {, anastrozole }+\mathrm{Gt} \text { vs. anastrozole }+ \\
\text { placebo }\end{array}$ & $\begin{array}{l}\text {-No added benefit, higher toxicity; } \\
\text { terminated prematurely }\end{array}$ \\
\hline
\end{tabular}

B, Erlotinib (Et)

\begin{tabular}{|c|c|c|c|c|}
\hline Dickler et al (23) & 2009 & II & $\begin{array}{l}\text {-ABC, unselected BC population, progression } \\
\text { under chemo }\end{array}$ & $\begin{array}{l}\text {-Minimal efficacy in unselected } \\
\text { population }\end{array}$ \\
\hline Lau et al (24) & 2014 & I & $-\mathrm{BBC}$, metformin $+\mathrm{Et}$ & -Increased apoptosis in a subset of BBC \\
\hline Ueno et al (25) & 2011 & I & -TNBC, xenograft model & $\begin{array}{l}\text {-Inhibition of metastasis, nonspecific } \\
\text { effects }\end{array}$ \\
\hline Guix et al (26) & 2008 & II & $-\mathrm{HR}^{+}$, stage I-IIIA & $\begin{array}{l}\text {-Inhibition of proliferation in } \mathrm{ER}^{+} \text {, not } \\
\text { in } \mathrm{HER}^{+} \text {or } \mathrm{TNBC}\end{array}$ \\
\hline
\end{tabular}

C, Afatinib (At)

\begin{tabular}{lllll} 
Harbeck et al (27) & 2016 & III & $\begin{array}{l}\text {-MBC, HER2 } 2^{+} \text {, progression on trastuzumab, } \\
\text { At + vinorelbine }\end{array}$ & $\begin{array}{l}\text {-Reduced efficacy of combination At }+ \\
\text { vinorelbine }\end{array}$ \\
Cortés et al (28) & 2015 & II & $\begin{array}{l}\text {-Brain MBC progressive or recurrent, HER2 }{ }^{+} \\
\text {-No additional benefit, frequent adverse } \\
\text { events }\end{array}$ \\
Hanusch et al (30) & 2015 & II & -ABC, At + trastuzumab, neoadjuvant & $\begin{array}{l}\text {-Comparable pCR with other anti- } \\
\text { HER2, but below expected }\end{array}$ \\
\hline
\end{tabular}

D, Lapatinib (Lt)

Baselga et al (32)

2012 III

-EBC, HER2 ${ }^{+}, \mathrm{Lt}$, and Lt + trastuzumab

2014 III

2014 II

2016 III

2006 III

2010 III

2010 III

2009 III

2010 III

de Azambuja et al (33) $2014 \quad$ III

Bonnefoi et al (34) 2015
-EBC, HER2 ${ }^{+}, \mathrm{Lt}, \mathrm{Lt}+$ trastuzumab

-ABC, HER2 $2^{+}$, neoadjuvant setting, Lt, Lt + trastuzumab, trastuzumab alone
-pCR significantly higher after Lt + trastuzumab vs. trastuzumab alone

-Event-free survival and OS did not differ between groups

-Modest pCR increase with anti-HER2 blockade (60\% vs. $52 \%$ ) 
Table I. Continued.

\begin{tabular}{|c|c|c|c|c|}
\hline First author (Refs.) & Year & Phase & Disease stage, regimen used & Outcome \\
\hline \multicolumn{5}{|l|}{ D, Lapatinib (Lt) } \\
\hline Piccart-Gebhart et al (35) & 2016 & III & $\begin{array}{l}\text {-EBC, HER } 2^{+} \text {, adjuvant setting, Lt, } \\
\text { Trastuzumab or combination }\end{array}$ & $\begin{array}{l}\text {-No improvement in DFS with Lt, but } \\
\text { added toxicity }\end{array}$ \\
\hline Geyer et al (36) & 2006 & III & $-\mathrm{ABC}, \mathrm{HER} 2^{+}, \mathrm{Lt}+$ capecitabine & $\begin{array}{l}-\mathrm{Lt}+\text { capecitabine was superior to } \\
\text { capecitabine alone }\end{array}$ \\
\hline Schwartzberg et al (37) & 2010 & III & $-\mathrm{MBC}, \mathrm{HER} 2^{+}, \mathrm{HR}^{+}, \mathrm{Lt}+$ letrozole & -Significantly higher PFS, ORR and CBR \\
\hline Sherrill et al (38) & 2010 & III & $-\mathrm{MBC}, \mathrm{HR}^{+}, \mathrm{HER}^{+}, \mathrm{Lt}+$ letrozole & $\begin{array}{l}\text {-Lt + letrozole increased PFS interval } \\
\text { compared with letrozole alone }\end{array}$ \\
\hline Johnston et al (39) & 2009 & III & -MBC $, \mathrm{HR}^{+}, \mathrm{HER} 2^{+}, 1$ st line therapy & $\begin{array}{l}\text {-Combined treatment significantly } \\
\text { enhanced PFS and CBR }\end{array}$ \\
\hline Blackwell et al (40) & 2010 & III & $-\mathrm{MBC}, \mathrm{HeR} 2^{+}, \mathrm{Lt}$ vs. $\mathrm{Lt}+$ trastuzumab & $\begin{array}{l}\text {-Combined treatment improved PFS and } \\
\text { CBR }\end{array}$ \\
\hline
\end{tabular}

E, Neratinib (Nt)

\begin{tabular}{|c|c|c|c|c|}
\hline Chow et al (42) & 2013 & $\mathrm{I} / \mathrm{II}$ & $-\mathrm{MBC}, \mathrm{HER} 2^{+}, \mathrm{Nt}+$ paclitaxel & -High rate of response, higher toxicity \\
\hline Chan et al (43) & 2016 & III & $\begin{array}{l}\text {-EBC/ABC, HER } 2^{+} \text {, adjuvant setting after } \\
\text { chemo and trastuzumab }\end{array}$ & $\begin{array}{l}\text {-Improvement of the DFS rate at the } \\
\text { 2-year follow up }\end{array}$ \\
\hline
\end{tabular}

$\mathrm{G}$, Canertinib $(\mathrm{Ct})$

\begin{tabular}{lllll}
\hline Rixe et al (44) & 2009 & II & -MBC, progressive or recurrent & -No clinically significant activity \\
\hline $\begin{array}{llll}\text { F, Tucatinib (Tt) } \\
\text { Murthy et al (45) }\end{array}$ & 2018 & Ib & -MBC, HER2 ${ }^{+}$, progressive BC & -Favorable antitumor activity, acceptable \\
Muthy et al (46) & 2020 & II & $\begin{array}{l}\text {-MBC, HER2 } \\
\text { toxicity }\end{array}$ \\
& & & with trastuzumab and capecitabine
\end{tabular}

H, Pyrotinib (Pt)

\begin{tabular}{|c|c|c|c|c|}
\hline Ma et al (47) & 2017 & I & -MBC, HER2 ${ }^{+}$ & $\begin{array}{l}\text {-Well tolerated, favorable antitumor } \\
\text { activity }\end{array}$ \\
\hline Ma et al (50) & 2019 & II & $\begin{array}{l}\text {-MBC, HER2 }{ }^{+} \text {, Pt combined with } \\
\text { capecitabine vs. lapatinib with capecitabine }\end{array}$ & $\begin{array}{l}\text {-Improved overall response rate and } \\
\text { PFS rate }\end{array}$ \\
\hline Jiang et al (51) & 2019 & III & $\begin{array}{l}\text {-MBC, HER2 } 2^{+}, \text {Pt combined with } \\
\text { capecitabine }\end{array}$ & $\begin{array}{l}\text {-Improved PFS; Pt monotherapy- } \\
\text { antitumor activity }\end{array}$ \\
\hline
\end{tabular}

$\mathrm{BC}$, breast cancer; PFS, progression-free survival; $\mathrm{TNBC}$, triple negative breast cancer; $\mathrm{EBC}$, early breast cancer; $\mathrm{ABC}$, advanced breast cancer; $\mathrm{MBC}$, metastatic breast cancer; $\mathrm{CBR}$, clinical benefit rate; $\mathrm{BBC}$, basal-like breast cancer; pCR, pathologic complete response rate; DFS, disease-free survival; OS, overall survival.

\section{Limitations and challenges of TKI use in BC chemotherapy}

The HER family of transmembrane receptors has been intensely studied due to its involvement in numerous aspects of $\mathrm{BC}$ development and progression. Optimal results were obtained for inhibition of HER2 overexpression with trastuzumab (Herceptin ${ }^{\circledR}$ ) and pertuzumab (Perjeta ${ }^{\circledR}$ ), which is now standard therapy in $\sim 20 \%$ of HER2-positive BC cases. HER2 blockade is used in neoadjuvant and adjuvant settings of incipient or locally advanced BC as well as in a metastatic setting.

For a few BC subtypes, for example TNBC, treatment options are quite limited. The lack of therapeutic targets makes 
subtypes such as TNBC or inflammatory BC difficult to treat with biological therapies. Actually, the only targeted therapy for TNBC is bevacizumab, a monoclonal antibody acting as an anti-VEGF inhibitor. However, in November 2011, the FDA revoked bevacizumab from the list of accelerated approvals for BC due to side effects and safety concerns (54).

Although targeted therapies lack cumulative bone marrow toxicity and other cytotoxic side effects of traditional chemotherapy, they do have a slightly different toxicity profile, involving mainly skin and gastrointestinal toxicities, including diarrhea (55). Rashes are usually pustular/papular and affect the upper half of the body. Improvement of diarrhea and skin rash toxicities is usually achieved with symptomatic medication. Severe cases of rash necessitate topical and systemic corticosteroids and antibiotics. Side effects are usually controlled using symptomatic medication, but occasionally dose reduction or dose interruption of anti-EGFR medication is required (55-57).

Interestingly, a positive correlation has been identified between skin toxicity/rash degree and tumor response. The correlation was found in two phase III clinical trials on NSCLC and pancreatic cancer, but did not correlate in BC studies (57).

For aggressive BC subtypes such as TNBC, combination of EGFR inhibition with other targeted therapies has provided encouraging results. Additive lethal interactions in vitro and in vivo were observed on $\mathrm{BC}$ cells for anti-EGFR lapatinib and poly (ADP-ribose) polymerase (PARP) inhibitor ABT-888 (veliparib). These findings could result in the widening of the indications for the use of PARP inhibitors beyond hereditary BRCA-mutated tumors, along with anti-EGFR agents, for TNBC (58). In addition to TNBC, the combination of an anti-EGFR agent and PARP inhibitor acted synergistically in HER2-enriched BC as well. PARP inhibitors olaparib and rucaparib increased the antitumor effects of trastuzumab in vitro and in vivo (59).

Immune-checkpoint inhibitors are novel targeted agents with broader spectrum of action, due to their capacity to block tumor-suppressive effects on activated T-cell lymphocytes (60). Animal studies revealed synergistic effects of trastuzumab and immune-checkpoint inhibitor, anti-PD-1, in immunocompetent mice (61). Trastuzumab and anti-PD-1 pembrolizumab are currently assessed in an ongoing phase I/II clinical trial with HER2-enriched metastatic BC patients (ClinicalTrials. gov: NCT02129556).

EGFR is one of the explored targets in BC with poorly expressed receptors, such as TNBC or inflammatory BC. However, difficulties in the selection of an appropriate subtype for anti-EGFR therapies has led to disappointing results. The mechanisms theorized for anti-EGFR treatment efficacy include the possibility to increase the sensitivity to cytotoxic agents and to prevent metastatic disease occurrence for aggressive BC subtypes. In a study of Ménard et al, an inverse correlation of hormone-receptor status and HER presence was documented, while HER2 heterodimers increased the metastatic potential of BC cells (62).

Identifying molecular subtypes of $\mathrm{BC}$ overexpressing the target under analysis, represents in fact the key of new target therapeutic strategy development. TNBC overexpresses EGFR in $50 \%$ of cases and thus appears to be a promising therapeutic target for biological agents (63). The difficulty in the methodology of selection of patients with activated EGFR vs. total EGFR expression represents an important task for the identification of the appropriate subset of patients who are responders to anti-EGFR therapy, based on an accurate evaluation of EGFR mutational status (64).

One of the positive effects of anti-EGFR-targeted agents is the chemo-sensitization property. Lee et al provided encouraging results of erlotinib and doxorubicin in TNBC with an increased rate of cancer cell death (65).

TNBC comprises a heterogeneous group of breast tumors incompletely characterized and separated from the other BC tumors only due to the lack of hormonal and HER2 receptors. An appropriate targeted therapeutic approach for TNBC will only be possible after improved subtype characterization and identification of predictive markers for response. Inflammatory BC overexpresses EGFR in $~ 30 \%$ to $40 \%$ of cases, associated with negative hormone receptors, which makes it an attractive target for biological agents $(66,67)$. However, application of anti-EGFR agents in these subtypes of BC still require considerable improvement until successful use in clinical practice. The discovery of a clinical agent until its use is a lengthy process (68).

The only anti-EGFR agent approved by the FDA for clinical use is lapatinib (Tyverb ${ }^{\circledR}$ ) in combination with chemotherapy or hormone therapy. It was revealed to be effective even in trastuzumab-resistant tumors although resistance occurrence is usually encountered and led researchers towards the development of irreversible second-generation pan-HER inhibitors, such as afatinib. However, the results were disappointing and no other EGFR inhibitor has made it to clinical use, to date.

Anti-EGFR targeted therapy has not yet overcome the benefits obtained from the inhibition of HER2 receptor with trastuzumab and pertuzumab, although blocking other HER receptors involved as well in cell signaling and proliferation was assumed to bring additional clinical benefits. Even the approval of lapatinib in clinical practice as an anti-HER1 and HER2 therapy was conducted only for HER2-positive $\mathrm{BC}$ and only in combination with additional concomitant therapy. However, additional targeted therapies including PARP inhibitors or immune-checkpoint inhibitors administered concomitantly with anti-EGFR agents may provide new insights in the treatment of aggressive BC subtypes.

\section{Conclusions}

HER2-positive BC is currently treated with monoclonal antibodies and small molecules of TKIs. TKIs have the advantage of low cardiotoxicity, oral administration, multiple cellular targets and increased crossing of the blood-brain barrier with positive therapeutic effects on $\mathrm{BC}$ brain metastases.

In clinical practice, lapatinib and neratinib are the only anti-EGFR agents approved by the FDA in clinical settings for the subset of HER2-enriched BC population, single or in association with other consecrated agents, while pyrotinib is approved by the Chinese State Drug Administration in combination with capecitabine in advanced or metastatic HER2-overexpressing BC.

Anti-EGFR therapy in BC requires improvement by identifying selected subtypes of tumors most susceptible to targeted therapy for optimal antitumor response and less toxicity. 
Whether this means treatment with single multi-kinase inhibitor or combined treatment with multiple single kinase inhibitors, is still a matter left to be answered on the basis of individualized treatment of each patient.

\section{Acknowledgements}

Not applicable.

\section{Funding}

No funding was received.

\section{Availability of data and materials}

All data generated or analyzed during this study are included in this published article.

\section{Authors' contributions}

GI, DV, CDB and RI conceived and designed the present study. GI, RI, MST, AZ, CTa were responsible for the data collection and analysis. DS, GI, CDB, AZ, DOC, CTu were in charge of drafting the manuscript. DS, MST, CTa, CTu, DOC, RI and DV revised the manuscript for important intellectual content. GI and RI confirm the authenticity of all the raw data. The final version was read and approved by all authors.

\section{Ethics approval and consent to participate}

Not applicable.

\section{Patient consent for publication}

Not applicable.

\section{Competing interests}

The authors declare that they have no competing interests.

\section{References}

1. Mendelsohn J and Baselga J: Status of epidermal growth factor receptor antagonists in the biology and treatment of cancer. J Clin Oncol 21: 2787-2799, 2003.

2. Harari PM: Epidermal growth factor receptor inhibition strategies in oncology. Endocr Relat Cancer 11: 689-708, 2004.

3. Moasser MM: The oncogene HER2: Its signaling and transforming functions and its role in human cancer pathogenesis Oncogene 26: 6469-6487, 2007.

4. Patel A, Unni N and Peng Y: The changing paradigm for the treatment of HER2-positive breast cancer. Cancers (Basel) 12: 2081, 2081, 2020.

5. Wuerstlein R and HarbeckN: Neoadjuvant therapy for HER2-positive breast cancer. Rev Recent Clin Trials 12: 81-92, 2017.

6. Reis-Filho JS and Tutt AN: Triple negative tumors: A critical review. Histopathology 52: 108-118, 2008.

7. Choi J, Jung WH and Koo JS: Clinicopathologic features of molecular subtypes of triple negative breast cancer based on immunohistochemical markers. Histol Histopathol 27: 1481-1493, 2012.

8. Martin V, Botta F, Zanellato E, Molinari F, Crippa S, Mazzucchelli L and Frattini M: Molecular characterization of EGFR and EGFR-downstream pathways in triple negative breast carcinomas with basal like features. Histol Histopathol 27: 785-792, 2012
9. Cai WQ, Zeng LS, Wang LF, Wang YY, Cheng JT, Zhang Y, Han ZW, Zhou Y, Huang SL, Wang XW, et al: The latest battles between EGFR monoclonal antibodies and resistant tumor cells. Front Oncol 10: 1249, 2020.

10. Rakha EA, El-Sayed ME, Green AR, Lee AH, Robertson JF and Ellis IO: Prognostic markers in triple-negative breast cancer. Cancer 109: 25-32, 2007.

11. Rosell R, Cardona AF, Arrieta O, Aguilar A, Ito M, Pedraz C, Codony-Servat $\mathbf{J}$ and Santarpia M: Coregulation of pathways in lung cancer patients with EGFR mutation: Therapeutic opportunities. Br J Cancer: 1-10, 2021 (Epub ahead of print).

12. Broekman F, Giovannetti E and Peters GJ: Tyrosine kinase inhibitors: Multi-targeted or single-targeted? World J Clin Oncol 2: 80-93, 2011.

13. Tetsu O, Hangauer MJ, Phuchareon J, Eisele DW and McCormick F: Drug resistance to EGFR inhibitors in lung cancer. Chemotherapy 61: 223-325, 2016.

14. He C, Li L, Guan X, Xiong L and Miao X: Mutant p53 gain of function and chemoresistance: The role of mutant p53 in response to clinical chemotherapy. Chemotherapy 62: 43-53, 2017.

15. Baselga J, Albanell J, Ruiz A, Lluch A, Gascón P, Guillém V, González S, Sauleda S, Marimón I, Tabernero JM, et al: Phase II and tumor pharmacodynamic study of gefitinib in patients with advanced breast cancer. J Clin Oncol 23: 5323-5333, 2005.

16. Green MD, Francis PA, Gebski V, Harvey V, Karapetis C, Chan A, Snyder R, Fong A, Basser R and Forbes JF; Australian New Zealand Breast Cancer Trials Group: Gefitinib treatment in hormone-resistant and hormone receptor-negative advanced breast cancer. Ann Oncol 20: 1813-1817, 2009.

17. Smith IE, Walsh G, Skene A, Llombart A, Mayordomo JI, Detre S, Salter J, Clark E, Magill P and Dowsett M: A phase II placebo-controlled trial of neoadjuvant anastrozole alone or with gefitinib in early breast cancer. J Clin Oncol 25: 3816-3822, 2007.

18. Polychronis A, Sinnett HD, Hadjiminas D, Singhal H, Mansi JL, Shivapatham D, Shousha S, Jiang J, Peston D, Barrett N, et al: Preoperative gefitinib versus gefitinib and anastrozole in postmenopausal patients with oestrogen-receptor positive and epid ermal-growth-factor-receptor-positive primary breast cancer: A double-blind placebo-controlled phase II randomised trial. Lancet Oncol 6: 383-391, 2005.

19. Cristofanilli M, Valero V, Mangalik A, Royce M, Rabinowitz I, Arena FP, Kroener JF, Curcio E, Watkins C, Bacus S, et al: Phase II, randomized trial to compare anastrozole combined with gefitinib or placebo in postmenopausal women with hormone receptor-positive metastatic breast cancer. Clin Cancer Res 16: 1904-1914, 2010.

20. Carlson RW, O'Neill A, Vidaurre T, Gomez HL, Badve SS and Sledge GW: A randomized trial of combination anastrozole plus gefitinib and of combination fulvestrant plus gefitinib in the treatment of postmenopausal women with hormone receptor positive metastatic breast cancer. Breast Cancer Res Treat 133: 1049-106, 2012.

21. Bernsdorf M, Ingvar C, Jörgensen L, Tuxen MK, Jakobsen EH, Saetersdal A, Kimper-Karl ML, Kroman N, Balslev E and Ejlertsen B: Effect of adding gefitinib to neoadjuvant chemotherapy in estrogen receptor negative early breast cancer in a randomized phase II trial. Breast Cancer Res Treat 126: 463-470, 2011.

22. Tryfonidis K, Basaran G, Bogaerts J, Debled M, Dirix L, Thery JC, Tjan-Heijnen VC, Van den Weyngaert D, Cufer T, Piccart M, et al: A European organisation for research and treatment of cancer randomized, double-blind, placebo-controlled, multicentre phase II trial of anastrozole in combination with gefitinib or placebo in hormone receptor-positive advanced breast cancer (NCT00066378). Eur J Cancer 53: 144-154, 2016.

23. Dickler MN, Cobleigh MA, Miller KD, Klein PM and Winer EP: Efficacy and safety of erlotinib in patients with locally advanced or metastatic breast cancer. Breast Cancer Res Treat 115: 115-121, 2009.

24. Lau YK, Du X, Rayannavar V, Hopkins B, Shaw J, Bessler E, Thomas T, Pires MM, Keniry M, Parsons RE, et al: Metformin and erlotinib synergize to inhibit basal breast cancer. Oncotarget 5: 10503-10517, 2014.

25. Ueno NT and Zhang D: Targeting EGFR in triple negative breast cancer. J Cancer 2: 324-228, 2011.

26. Guix M, Granja Nde M, Meszoely I, Adkins TB, Wieman BM, Frierson KE, Sanchez V, Sanders ME, Grau AM, Mayer IA, et al: Short preoperative treatment with erlotinib inhibits tumor cell proliferation in hormone receptor-positive breast cancers. J Clin Oncol 26: 897-906, 2008. 
27. Harbeck N, Huang CS, Hurvitz S, Yeh DC, Shao Z, Im SA, Jung KH, Shen K, Ro J, Jassem J, et al: Afatinib plus vinorelbine versus trastuzumab plus vinorelbine in patients with HER2-overexpressing metastatic breast cancer who had progressed on one previous trastuzumab treatment (LUX-Breast 1): An open-label, randomised, phase 3 trial. Lancet Oncol 17: 357-366, 2016.

28. Cortés J, Dieras V, Ro J, Barriere J, Bachelot T, Hurvitz S, Le Rhun E, Espié M, Kim SB, Schneeweiss A, et al: Afatinib alone or afatinib plus vinorelbine versus investigator's choice of treatment for HER2-positive breast cancer with progressive brain metastases after trastuzumab, lapatinib, or both (LUX-Breast 3): A randomised, open-label, multicentre, phase 2 trial. Lancet Oncol 16: 1700-1710, 2015

29. Kawaguchi Y, Hanaoka J, Hayashi H, Mizusaki N, Iihara H, Itoh Y and Sugiyama T: Clinical efficacy of afatinib treatment for a patient with leptomeningeal carcinomatosis. Chemotherapy 62: 147-150, 2017.

30. Hanusch C, Schneeweiss A, Loibl S, Untch M, Paepke S, Kümmel S, Jackisch C, Huober J, Hilfrich J, Gerber B, et al: Dual blockade with afatinib and trastuzumab as NEoadjuvant treatment for patients with locally advanced or operable breast cancer receiving taxane-anthracycline containing chemotherapy-DAFNE (GBG-70). Clin Cancer Res 21: 2924-291, 2015.

31. Chen ZL, Shen YW, Li ST, Li CL, Zhang LX, Yang J, Lv M, Lin YY, Wang X and Yang J: The efficiency and safety of trastuzumab and lapatinib added to neoadjuvant chemotherapy in Her2-positive breast cancer patients: A randomized meta-analysis. Onco Targets Ther 9: 3233-3247, 2016.

32. Baselga J, Bradbury I, Eidtmann H, Di Cosimo S, de Azambuja E, Aura C, Gómez H, Dinh P, Fauria K, Van Dooren V, et al: Lapatinib with trastuzumab for HER2-positive early breast cancer (NeoALTTO): A randomised, open-label, multicentre, phase 3 trial. Lancet 379: 633-640, 2012.

33. de Azambuja E, Holmes AP, Piccart-Gebhart M, Holmes E, Di Cosimo S, Swaby RF, Untch M, Jackisch C, Lang I, Smith I, et al: Lapatinib with trastuzumab for HER2-positive early breast cancer (NeoALTTO): Survival outcomes of a randomised, open-label, multicentre, phase 3 trial and their association with pathological complete response. Lancet Oncol 15 $1137-1146,2014$

34. Bonnefoi H, Jacot W, Saghatchian M, Moldovan C, Venat-Bouvet L, Zaman K, Matos E, Petit T, Bodmer A, Quenel-Tueux N, et al: Neoadjuvant treatment with docetaxel plus lapatinib, trastuzumab, or both followed by an anthracycline-based chemotherapy in HER2-positive breast cancer: Results of the randomised phase II EORTC 10054 study. Ann Oncol 26: 325-332, 2015

35. Piccart-Gebhart M, Holmes E, Baselga J, de Azambuja E, Dueck AC, Viale G, Zujewski JA, Goldhirsch A, Armour A, Pritchard KI, et al: Adjuvant lapatinib and trastuzumab for early human epidermal growth factor receptor 2-positive breas cancer: Results from the randomized phase III adjuvant lapatinib and/or trastuzumab treatment optimization trial. J Clin Oncol 34 1034-1042, 2016

36. Geyer CE, Forster J, Lindquist D, Chan S, Romieu CG, Pienkowski T, Jagiello-Gruszfeld A, Crown J, Chan A, Kaufman B, et al: Lapatinib plus capecitabine for HER2-positive advanced breast cancer. N Engl J Med 355: 2733-2743, 2006

37. Schwartzberg LS, Franco SX, Florance A, O'Rourke L, Maltzman J and Johnston S: Lapatinib plus letrozole as first-line therapy for HER-2+ hormone receptor-positive metastatic breast cancer. Oncologist 15: 122-129, 2010.

38. Sherrill B, Amonkar MM, Sherif B, Maltzman J, O'Rourke L and Johnston S: Quality of life in hormone receptor-positive HER-2+ metastatic breast cancer patients during treatment with letrozole alone or in combination with lapatinib. Oncologist 15: 944-953, 2010.

39. Johnston S, Pippen J Jr, Pivot X, Lichinitser M, Sadeghi S Dieras V, Gomez HL, Romieu G, Manikhas A, Kennedy MJ, et al: Lapatinib combined with letrozole versus letrozole and placebo as first-line therapy for postmenopausal hormone receptor-positive metastatic breast cancer. J Clin Oncol 27: 5538-5546, 2009.

40. Blackwell KL, Burstein HJ, Storniolo AM, Rugo H, Sledge G Koehler M, Ellis C, Casey M, Vukelja S, Bischoff J, et al: Randomized study of lapatinib alone or in combination with trastuzumab in women with ErbB2-positive, trastuzumab-refractory metastatic breast cancer. J Clin Oncol 28: 1124-1130, 2010.
41. National Comprehensive Cancer Network. NCCN clinical practice guidelines in oncology (NCCN Guidelines $\left.{ }^{\circledR}\right)$ : Breast cancer version 2.2016, 2016

42. Chow LW, Xu B, Gupta S, Freyman A, Zhao Y, Abbas R, Vo Van ML and Bondarenko I: Combination neratinib (HKI-272) and paclitaxel therapy in patients with HER2-positive metastatic breast cancer. Br J Cancer 108: 1985-1993, 2013.

43. Chan A, Delaloge S, Holmes FA, Moy B, Iwata H, Harvey VJ, Robert NJ, Silovski T, Gokmen E, von Minckwitz G, et al: Neratinib after trastuzumab-based adjuvant therapy in patients with HER2-positive breast cancer (ExteNET): A multicentre, randomised, double-blind, placebo-controlled, phase 3 trial. Lancet Oncol 17: 367-377, 2016.

44. Rixe O, Franco SX, Yardley DA, Johnston SR, Martin M, Arun BK, Letrent SP and Rugo HS: A randomized, phase II, dose-finding study of the pan-ErbB receptor tyrosine-kinase inhibitor CI-1033 in patients with pretreated metastatic breast cancer. Cancer Chemother Pharmacol 64: 1139-1148, 2009.

45. Murthy R, Borges VF, Conlin A, Chaves J, Chamberlain M, Gray T, Vo A and Hamilton E: Tucatinib with capecitabine and trastuzumab in advanced HER2-positive metastatic breast cancer with and without brain metastases: A non-randomised, open-label, phase 1b study. Lancet Oncol 19: 880-888, 2018.

46. Murthy RK, Loi S, Okines A, Paplomata E, Hamilton E, Hurvitz SA, Lin NU, Borges V, Abramson V, Anders C, et al: Tucatinib, trastuzumab, and capecitabine for HER2-positive metastatic breast cancer. N Engl J Med 382: 597-609, 2020.

47. Ma F, Li Q, Chen S, Zhu W, Fan Y, Wang J, Luo Y, Xing P, Lan B, Li M, et al: Phase I study and biomarker analysis of pyrotinib, a novel irreversible pan-ErbB receptor tyrosine kinase inhibitor, in patients with human epidermal growth factor receptor 2-positive metastatic breast cancer. J Clin Oncol 35: 3105-3112, 2017.

48. Zhang K, Hong R, Kaping L, Xu F, Xia W, Qin G, Zheng Q, Lu Q, Zhai Q, Shi Y, et al: CDK4/6 inhibitor palbociclib enhances the effect of pyrotinib in HER2-positive breast cancer. Cancer Lett 447: 130-140, 2019.

49. Xuhong JC, Qi XW, Zhang Y and Jiang J: Mechanism, safety and efficacy of three tyrosine kinase inhibitors lapatinib, neratinib and pyrotinib in HER2-positive breast cancer. Am J Cancer Res 9: 2103-2119, 2019.

50. Ma F, Ouyang Q, Li W, Jiang Z, Tong Z, Liu Y, Li H, Yu S, Feng J, Wang S, et al: Pyrotinib or lapatinib combined with capecitabine in HER2-positive metastatic breast cancer with prior taxanes, anthracyclines, and/or trastuzumab: A randomized, phase II study. J Clin Oncol 37: 2610-2619, 2019.

51. Jiang Z, Yan M, Hu X, Zhang Q, Ouyang Q, Feng J, Yin Y, Sun T, Tong Z, Wang X, et al: Pyrotinib combined with capecitabine in women with HER2+ metastatic breast cancer previously treated with trastuzumab and taxanes: A randomized phase III study. J Clin Oncol 37 (Suppl 15): S1001, 2019.

52. He L, Zhang F, Ma Y, Zuo L and Xu Y: Pathological complete response from pyrotinib combined with trastuzumab, paclitaxel and cisplatin in a postpartum woman with HER2-positive locally advanced breast cancer: A case report. Onco Targets Ther 13: 8749-8756, 2020

53. Wang C, Lin Y, Zhou Y, Mao F, Zhu H, Guan J, Zhang X, Shen S, Huang X, Chen C, et al: Pyrotinib with trastuzumab and aromatase inhibitors as first-line treatment for HER2 positive and hormone receptor positive metastatic or locally advanced breast cancer: Study protocol of a randomized controlled trial. BMC Cancer 20: 653, 2020.

54. Goozner M: Avastin hearing leads to more uncertainty over drug's future. J Natl Cancer Inst 103: 1148-1150, 2011

55. Barton-Burke M, Ciccolini K, Mekas $M$ and Burke S: Dermatologic reactions to targeted therapy: A focus on epidermal growth factor receptor inhibitors and nursing care. Nurs Clin North Am 52: 83-113, 2017.

56. Melosky B and Hirsh V: Management of common toxicities in metastatic NSCLC related to anti-lung cancer therapies with EGFR-TKIs. Front Oncol 4: 238, 2014.

57. Wacker B, Nagrani T, Weinberg J, Witt K, Clark G and Cagnoni PJ: Correlation between development of rash and efficacy in patients treated with the epidermal growth factor receptor tyrosine kinase inhibitor erlotinib in two large phase III studies. Clin Cancer Res 13: 3913-3921, 2007.

58. Nowsheen S, Cooper T, Stanley JA and Yang ES: Synthetic lethal interactions between EGFR and PARP inhibition in human triple negative breast cancer cells. PLoS One 7: e46614, 2012. 
59. García-Parra J, Dalmases A, Morancho B, Arpí O, Menendez S, Sabbaghi M, Zazo S, Chamizo C, Madoz J, Eroles P, et al: Poly (ADP-ribose) polymerase inhibition enhances trastuzumab antitumour activity in HER2 overexpressing breast cancer. Eur J Cancer 50: 2725-2734, 2014.

60. Topalian SL, Hodi FS, Brahmer JR, Gettinger SN, Smith DC, McDermott DF, Powderly JD, Carvajal RD, Sosman JA, Atkins MB, et al: Safety, activity, and immune correlates of anti-PD-1 antibody in cancer. N Engl J Med 366: 2443-2454, 2012.

61. Stagg J, Loi S, Divisekera U, Ngiow SF, Duret H, Yagita H, Teng MW and Smyth MJ: Anti-ErbB-2 mAb therapy requires type I and II interferons and synergizes with anti-PD-1 or anti-CD137 mAb therapy. Proc Natl Acad Sci USA 108: 7142-7147, 2011

62. Ménard S, Balsari A, Casalini P, Tagliabue E, Campiglio M, Bufalino R and Cascinelli N: HER-2-positive breast carcinomas as a particular subset with peculiar clinical behaviors. Clin Cancer Res 8: 520-525, 2002.

63. Dent R, Trudeau M, Pritchard KI, Hanna WM, Kahn HK, Sawka CA, Lickley LA, Rawlinson E, Sun P and Narod SA: Triple-negative breast cancer: Clinical features and patterns of recurrence. Clin Cancer Res 13: 4429-4434, 2007.
64. Hoeijmakers JH: Genome maintenance mechanisms for preventing cancer. Nature 411: 366-374, 2001

65. Lee MJ, Ye AS, Gardino AK, Heijink AM, Sorger PK, MacBeath G and Yaffe MB: Sequential application of anticancer drugs enhances cell death by rewiring apoptotic signaling networks. Cell 149: 780-794, 2012.

66. Dawood S, Merajver SD, Viens P, Vermeulen PB, Swain SM, Buchholz TA, Dirix LY, Levine PH, Lucci A Krishnamurthy $\mathrm{S}$, et al: International expert panel on inflammatory breast cancer: Consensus statement for standardized diagnosis and treatment. Ann Oncol 22: 515-523, 2011.

67. Zell JA, Tsang W Y, Taylor TH, Mehta RS and Anton-Culver H: Prognostic impact of human epidermal growth factor-like receptor 2 and hormone receptor status in inflammatory breast cancer (IBC): Analysis of 2,014 IBC patient cases from the California cancer registry. Breast Cancer Res 11: R9, 2009.

68. Nitulescu GM, Margina D, Juzenas P, Peng Q, Olaru OT, Saloustros E, Fenga C, Spandidos DA, Libra $M$ and Tsatsakis AM: Akt inhibitors in cancer treatment: The long journey from drug discovery to clinical use (Review). Int $\mathbf{J}$ Oncol 48: 869-885, 2016. 